BRAVZILILN JOURNAL

OF MEDICAL AND BIOLOGICAL RESH.ARCH

www.bjournal.com.br
ISSN 0100-879X

Volume 43 (12) 1135-1244 December 2010

BIOMEDICAL SCIENCES

AND

CLINICAL INVESTIGATION

Braz J Med Biol Res, December 2010, Volume 43(12) 1173-1177

doi: 10.1590/S0100-879X2010007500130

Increased levels of glutamate in the central nervous system are associated with behavioral symptoms in experimental malaria

A.S. Miranda, L.B. Vieira, N. Lacerda-Queiroz, A.H. Souza, D.H. Rodrigues, M.C. Vilela, M.V. Gomez, F.S. Machado, M.A. Rachid and A.L. Teixeira

The Brazilian Journal of Medical and Biological Research is partially financed by
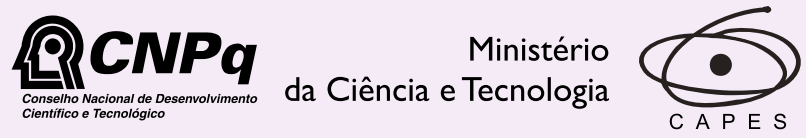

Ministério da Educação



DFAPESP

Institutional Sponsors
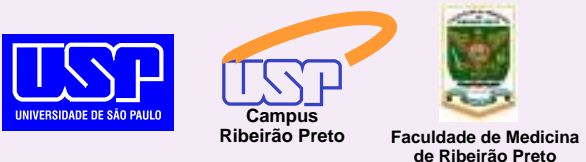

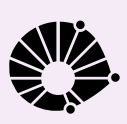

UNICAMP
Ф SHIMADZU

GE Healthcare
Hotsite of proteomics metabolomics developped by:

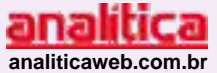

Thermo
SCIEN TIFIC 


\title{
Increased levels of glutamate in the central nervous system are associated with behavioral symptoms in experimental malaria
}

\author{
A.S. Miranda ${ }^{1}$, L.B. Vieira ${ }^{3}$, N. Lacerda-Queiroz ${ }^{1}$, A.H. Souza ${ }^{3}$, D.H. Rodrigues ${ }^{1}$, \\ M.C. Vilela1 ${ }^{1}$, M.V. Gomez ${ }^{3}$, F.S. Machado ${ }^{2}$, M.A. Rachid ${ }^{1}$ and A.L. Teixeira ${ }^{1}$ \\ ${ }^{1}$ Laboratório de Imunofarmacologia, ${ }^{2}$ Laboratório de Imunorregulação de Doenças Infecciosas, \\ Departamento de Bioquímica e Imunologia, Instituto de Ciências Biológicas, \\ Universidade Federal de Minas Gerais, Belo Horizonte, MG, Brasil \\ ${ }^{3}$ Laboratório de Medicina Molecular (INCT), Faculdade de Medicina, \\ Universidade Federal de Minas Gerais, Belo Horizonte, MG, Brasil
}

\begin{abstract}
Cerebral malaria (CM) is a severe complication resulting from Plasmodium falciparum infection. This condition has been associated with cognitive, behavioral and motor dysfunctions, seizures and coma. The underlying mechanisms of CM are incompletely understood. Glutamate and other metabolites such as lactate have been implicated in its pathogenesis. In the present study, we investigated the involvement of glutamate in the behavioral symptoms of CM. Seventeen female C57BL/6 mice (20-25 g) aged 6-8 weeks were infected with $P$. berghei ANKA by the intraperitoneal route using a standardized inoculation of $10^{6}$ parasitized red blood cells suspended in $0.2 \mathrm{~mL}$ PBS. Control animals $(\mathrm{N}=17)$ received the same volume of PBS. Behavioral and neurological symptoms were analyzed by the SmithKline/Harwell/Imperial College/Royal Hospital/Phenotype Assessment (SHIRPA) battery. Glutamate release was measured in the cerebral cortex and cerebrospinal fluid of infected and control mice by fluorimetric assay. All functional categories of the SHIRPA battery were significantly altered in the infected mice at 6 days post-infection (dpi) $(\mathrm{P} \leq 0.05)$. In parallel to $\mathrm{CM}$ symptoms, we found a significant increase in glutamate levels in the cerebral cortex (mean \pm SEM; control: $11.62 \pm 0.90 \mathrm{nmol} / \mathrm{mg}$ protein; infected at $3 \mathrm{dpi}: 10.36 \pm 1.17 \mathrm{nmol} / \mathrm{mg}$ protein; infected at $6 \mathrm{dpi}$ : $26.65 \pm 0.73 \mathrm{nmol} / \mathrm{mg}$ protein; with EGTA, control: $5.60 \pm 1.92 \mathrm{nmol} / \mathrm{mg}$ protein; infected at $3 \mathrm{dpi}: 6.24 \pm 1.87 \mathrm{nmol} / \mathrm{mg} \mathrm{protein}$; infected at $6 \mathrm{dpi}: 14.14 \pm 0.84 \mathrm{nmol} / \mathrm{mg}$ protein) and in the cerebrospinal fluid (control: $128 \pm 51.23 \mathrm{pmol} / \mathrm{mg} \mathrm{protein}$; infected: $301.4 \pm 22.52 \mathrm{pmol} / \mathrm{mg}$ protein) of infected mice $(\mathrm{P} \leq 0.05)$. These findings suggest a role of glutamate in the central nervous system dysfunction found in CM.
\end{abstract}

Key words: Cerebral malaria; Glutamate; Cerebrospinal fluid; Behavioral changes; SHIRPA

\section{Introduction}

Cerebral malaria $(\mathrm{CM})$ is a severe complication resulting from Plasmodium falciparum infection (1). This condition is associated with at least 2.3 million deaths per year out of an estimated 400 million cases of malaria occurring each year worldwide (2).

According to WHO criteria, CM is a clinical syndrome defined as a potentially reversible diffuse encephalopathy characterized mainly by coma and the presence of asexual forms of $P$. falciparum parasites in peripheral blood smears in the absence of other causes of encephalopathy (3). This condition can cause a wide range of clinical manifestations including cognitive, behavioral and motor dysfunctions, seizures and coma (1).

The underlying mechanisms of $\mathrm{CM}$ have been extensively investigated. However, the pathogenesis of $\mathrm{CM}$ is incompletely understood (4). One of the major hypotheses is the sequestration of parasitized red blood cells in the cerebral microvascular endothelium leading to blood flow obstruction and decreased tissue perfusion, thereby compromising the function of the central nervous system (CNS) (1).

Glutamate is the major excitatory neurotransmitter in

Correspondence: A.S. Miranda and A.L. Teixeira, Laboratório de Imunofarmacologia, Departamento de Bioquímica e Imunologia, ICB, UFMG, Av. Antônio Carlos, 6627, 31270-901 Belo Horizonte, MG, Brasil. Fax: +55-31-3409-2651.

E-mail: alines.miranda@hotmail.com and altexr@gmail.com

Received June 2, 2010. Accepted October 27, 2010. Available online November 19, 2010. Published December 20, 2010. 
the mammalian CNS, playing an important role in neuronal development, synaptic plasticity, learning and memory processes under physiological conditions (5). However, high amounts of glutamate release in intersynaptic spaces can cause neuronal cell death and neurodegeneration via excitotoxicity processes (6). Excitotoxicity plays an important role in many CNS diseases, including ischemia, trauma, and neurodegenerative disorders (7). Based on the concept that $\mathrm{CM}$ can be regarded as an ischemic disorder, some studies have implicated glutamate and other metabolites such as lactate, alanine and glycine in its pathogenesis (8-10). However, these studies did not evaluate the role of glutamate release and its association with CNS dysfunction in CM.

In the present study, we determined the involvement of glutamate in the behavioral symptoms occurring in CM. We analyzed behavioral and neurological symptoms, $\mathrm{Ca}^{2+}$-independent and-dependent glutamate release in the cerebral cortex and glutamate levels in the cerebrospinal fluid (CSF) of C57BL/6 mice infected with P. berghei ANKA (PbA).

\section{Material and Methods}

\section{Animals}

Thirty-four female C57BL/6 mice (20-25 g) aged 6-8 weeks were obtained from the Animal Care Facilities of the Federal University of Minas Gerais, Brazil. The animals were housed in cages in temperature-controlled rooms and received food and water ad libitum. All procedures described had prior approval from the Animal Ethics Committee (CETEA) of the Federal University of Minas Gerais (UFMG) under license number 105/09.

\section{Parasites and experimental infection}

An uncloned parasite line of $P$. berghei (strain ANKA) $(\mathrm{PbA})$ was used. $P$. bergheiANKA-parasitized red blood cells ( $p R B C$ ) from $C 57 B L / 6$ mouse donor strains were maintained in stabilized liquid nitrogen, thawed and passed into normal C57BL/6 mice that later served as parasite donors. C57BL/6 mice were infected by intraperitoneal (ip) injection of $10^{6}$ pRBC suspended in $0.2 \mathrm{~mL}$ PBS (11). Control animals received the same volume of PBS. The level of parasitemia of infected mice was monitored on Giemsa-stained blood films from day 3 onwards and estimated by counting at least 1000 RBCs under oil immersion.

\section{SHIRPA screen}

Behavioral and functional parameters were evaluated using a screening battery called SmithKline/Harwell/Imperial College/Royal Hospital/Phenotype Assessment (SHIRPA) until the 6th day post-infection (dpi). The procedure was carried out at 0 (day of infection) and then from day 3 until death on a daily basis. The SHIRPA screen was conceived as a multi-test behavioral battery used for longitudinal studies with standardized guidelines and materials (12). This battery encompasses 40 tests, which provide a behavioral and functional profile. For analysis purposes, these individual parameters assessed by SHIRPA were organized into five functional categories: neuropsychiatric state (spontaneous activity, transfer arousal, touch escape, positional passivity, biting, fear, irritability, aggression, vocals); motor behavior (body position, tremor, locomotor activity, pelvic elevation, gait, tail elevation, trunk curl, limb grasping, wire maneuver, negative geotaxis); reflex and sensory function (startle response, visual placing, pinna reflex, corneal reflex, toe pinch, righting reflex); autonomous function (respiration rate, defecation, urination, palpebral closure, piloerection, skin color, heart rate, lacrimation, salivation, body temperature); muscle tone and strength (grip strength, body tone, limb tone, abdominal tone) and an overall score was obtained as described by Lackner et al. (13). Animals were allowed to habituate to their new environment for 2 days before behavioral assessment. A total of 8 animals per group were used in this procedure.

\section{Glutamate release and measurement in the cerebral cortex}

Synaptosomes were prepared as previously described (14). Mice were decapitated and their cortices were removed and homogenized in 1:10 (w/v) $0.32 \mathrm{M}$ sucrose containing $0.25 \mathrm{mM}$ dithiothreitol and $1 \mathrm{mM}$ EDTA. Homogenates were then submitted to low-speed centrifugation (1000 g/10 min) and isolated nerve terminals (synaptosomes) were purified from the supernatant by discontinuous Percolldensity gradient centrifugation (15). The synaptosomes were resuspended in $400 \mu \mathrm{L}$ Krebs-Ringer-HEPES buffer (124 mM NaCl, 4 mM KCl, $1.2 \mathrm{mM} \mathrm{MgSO}_{4}, 10 \mathrm{mM}$ glucose, 25 mM HEPES, pH 7.4), divided into 200- $\mu$ L aliquots and stored on ice for later measurement of glutamate release. The glutamate release assay was performed using an RF5301PC spectrofluorimeter (Shimadzu, Japan) monitoring the increase in fluorescence due to the production of $\mathrm{NADPH}^{+}$in the presence of glutamate dehydrogenase and $\mathrm{NADP}^{+}(16)$. Glutamate release was measured in the cerebral cortex of PbA-infected mice at 3 and 6 dpi and of control mice. A total of 9 animals per group were used.

\section{Measurements of glutamate levels in the cerebrospinal fluid}

Glutamate levels were measured in the CSF of PbAinfected mice at $6 \mathrm{dpi}$ and of control mice. Briefly, the mice were killed with halothane and placed in a stereotaxic apparatus, where the CSF was carefully removed (10 $\mu \mathrm{L}$ per mouse) with an insulin syringe (27 gauge $\times 1 / 2$ in length), using the cisterna magna puncture technique. All samples were centrifuged at 10,000 $\mathrm{g}$ in an Eppendorf centrifuge for $5 \mathrm{~min}$ to obtain cell-free supernatants and were immediately analyzed with a spectrofluorimeter. Glutamate measurement was performed enzymatically according 
to the increase in fluorescence due to the production of $\mathrm{NADPH}^{+}$in the presence of glutamate dehydrogenase and $\mathrm{NADP}^{+}$(16). To start the assay, $1.0 \mathrm{mM} \mathrm{NADP}^{+}$and $50 \mathrm{U}$ glutamate dehydrogenase were added to the CSF samples 10 min after the measurement of emitted fluorescence (14). The excitation wavelength was $360 \mathrm{~nm}$ and the emission wavelength was $450 \mathrm{~nm}$ using a PTI spectrofluorimeter. Nine animals per group were used to quantify the levels of glutamate in the CSF. At least three independent experiments were performed and three samples of the CSF were analyzed in each group. The samples were obtained from the same animals used for glutamate measurements in the cerebral cortex.

\section{Statistical analysis}

One-way analysis of variance (ANOVA) with the Tukey multiple comparison post-test was used to analyze the behavioral and functional categories of SHIRPA and the brain cortical glutamate release. The unpaired Student $t$-test was used to analyze glutamate levels in the CSF. All analyses were performed using the Prism 4 software (GraphPad, USA).

\section{Results}

The SHIRPA battery was used to evaluate the behavioral changes of infected mice at 3 and $6 \mathrm{dpi}$. No difference was found between infected mice and controls at $3 \mathrm{dpi}$. However, all functional categories of the SHIRPA battery were significantly altered in infected mice at 6 dpi compared to the control group. When the infected mice were compared at 6 dpi to the infected mice at $3 \mathrm{dpi}$, a progressive impairment of autonomous function, neuropsychiatric state, motor behavior and muscle tone and strength was observed (Figure 1).

Since abnormal glutamatergic neurotransmission has been implicated in a wide range of neurological diseases, we measured glutamate release from isolated brain cortical nerve terminals (synaptosomes) in this CM model. No difference was found between infected mice at 3 dpi and controls. We observed that glutamate release was significantly increased in the infected mice at $6 \mathrm{dpi}$ in comparison with both infected mice at $3 \mathrm{dpi}$ and control animals (Figure 2). Synaptosomes from control animals were exposed to $33 \mathrm{mM}$ $\mathrm{KCl}$ to depolarize their membranes and induce glutamate release (Figure 2). $\mathrm{KCl}$-evoked glutamate release from synaptosomes obtained from infected mice at 6 dpi was approximately 3 -fold higher than that observed in control animals (Figure $2 ; \mathrm{P} \leq 0.05$ ).

When synaptosomes were depolarized with $\mathrm{KCl}$, the release of glutamate was the sum of two components: one that is extracellular calcium dependent and inhibited by the calcium chelator EGTA, and the other, which is extracellular calcium independent and not sensitive to EGTA. We therefore measured $\mathrm{KCl}$-evoked glutamate release in infected mice at 3 and 6 dpi and in control synaptosomes in the presence of EGTA, which reflects the calcium-independent pool. In both situations (control and infected mice), $\mathrm{KCl}$ evoked glutamate release was reduced in the presence of EGTA (Figure 2; $P \leq 0.05$ ). Even in the presence of EGTA,
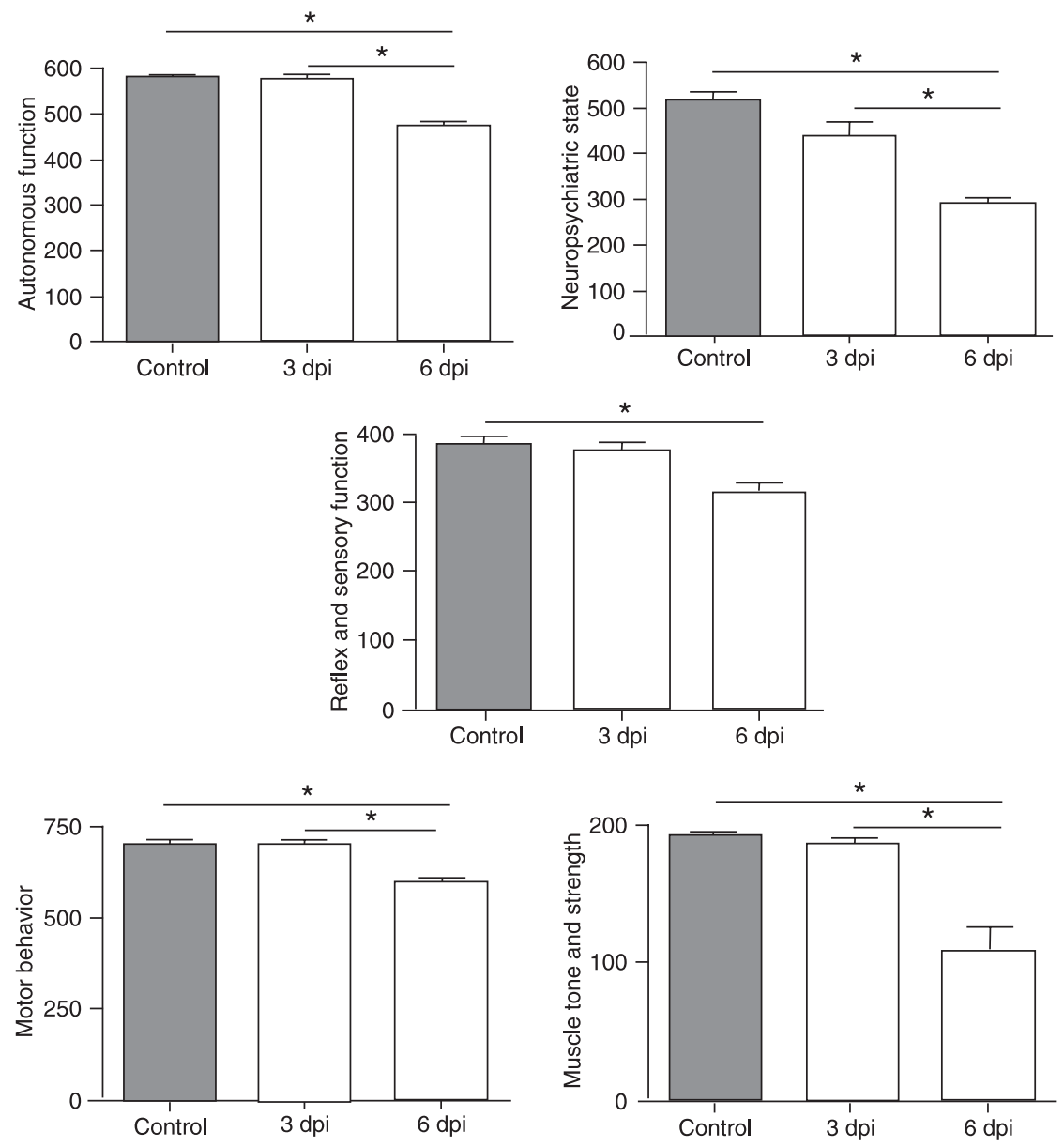

Figure 1. Performance of Plasmodium berghei ANKA (PbA)-infected mice at 3 and 6 days post-infection (dpi) and of the control group in the five functional categories of the SHIRPA battery. An overall score was used for each functional domain. Data are reported as means \pm SEM. ${ }^{*} \mathrm{P} \leq 0.05$ (ANOVA followed by the Tukey multiple comparison post-test). 
glutamate release was significantly increased in infected mice at 6 dpi compared to both infected mice at 3 dpi and controls.

We also measured CSF glutamate levels in $\mathrm{PbA}$-infected mice at $6 \mathrm{dpi}$. The 6 th dpi was chosen because it was the day we found the highest levels of brain glutamate associated with behavioral changes in infected mice. The glutamate levels found in the CSF of infected mice were significantly increased compared to control animals (mean \pm SEM; control: $128 \pm 51.23 \mathrm{pmol} / \mathrm{mg}$ protein; infected: $301.4 \pm 22.52$ $\mathrm{pmol} / \mathrm{mg}$ protein; $\mathrm{P} \leq 0.05$. Results are representative of three independent experiments).

\section{Discussion}

We investigated behavioral symptoms in C57BL/6 mice infected with $\mathrm{PbA}$ using the SHIRPA screen battery. In our study, we observed progressive neurological and behavioral changes. At 6 dpi most of the SHIRPA domains were significantly altered including, neuropsychiatric state, motor behavior, reflex and sensory function, autonomous function, muscle tone and strength. In order to detect a neurochemical marker of $\mathrm{CM}$, we investigated the involvement of glutamate in the development of $\mathrm{CM}$. The amount of glutamate present in the cerebrocortical synaptosomes as well as in the CSF of infected mice was significantly increased at $6 \mathrm{dpi}$. Since there is a parallel increase in brain and CSF glutamate levels with the neurological symptoms of CM, this may suggest a role for glutamate in $\mathrm{CM}$ pathogenesis.

Animals susceptible to $\mathrm{PbA}$ infection such as $\mathrm{C} 57 \mathrm{BL} / 6$ mice develop neurological and behavioral symptoms that are similar to those observed in human $\mathrm{CM}$, which include ataxia, paralysis, seizures, and coma (17). In the present study, using the SHIRPA battery, we found a wide range of behavioral changes in infected mice at $6 \mathrm{dpi}$. In agreement with these findings, previous studies also described significant changes in the functional categories of the SHIRPA battery approximately 6 days after $\mathrm{PbA}$ infection $(13,18)$.

The excitotoxicity process mediated by glutamate and other amino acids, such as aspartic acid and quinolinic acid that act via glutamate receptors, has been implicated in the occurrence of neurological and cognitive symptoms in CM $(8-10,19,20)$. In the present study, we found increased glutamate release in the brain and increased levels of this neurotransmitter in the CSF of $\mathrm{PbA}$-infected mice in association with behavioral changes. To the best of our knowledge, no previous study has investigated the association between increased glutamate release into intersynaptic spaces and CNS dysfunction in CM. A study performed by Rae et al.

\section{References}

1. Idro R, Jenkins NE, Newton CR. Pathogenesis, clinical features, and neurological outcome of cerebral malaria. Lancet Neurol 2005; 4: 827-840.

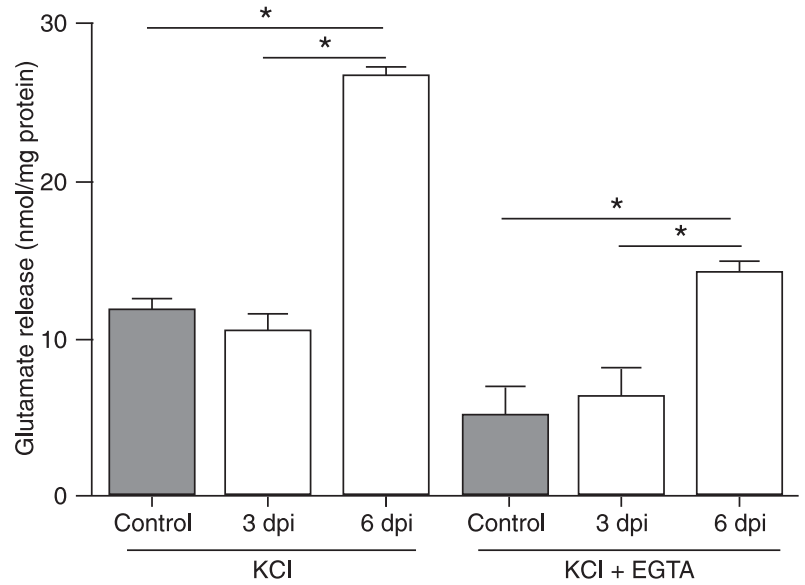

Figure 2. Cerebral malaria induces glutamate release from brain synaptosomes. $\mathrm{KCl}: 33 \mathrm{mM} \mathrm{KCl-evoked} \mathrm{glutamate} \mathrm{release} \mathrm{from}$ C57BL/6 control mice and from C57BL/6 Plasmodium berghei ANKA (PbA)-infected mice at 3 and 6 days post-infection (dpi). $\mathrm{KCl}+\mathrm{EGTA}$ : Calcium-independent glutamate release evoked by $33 \mathrm{mM} \mathrm{KCl}$ from C57BL/6 control mice synaptosomes and from $\mathrm{C} 57 \mathrm{BL} / 6 \mathrm{PbA}$-infected mice at 3 and $6 \mathrm{dpi}$. Data are reported as means \pm SEM of at least three independent experiments for each experimental condition. ${ }^{*} \mathrm{P} \leq 0.05$ (ANOVA followed by the Tukey multiple comparison post-test).

(9) demonstrated an increase of glutamate C4(Y) levels as measured by ${ }^{13} \mathrm{C}$ nuclear magnetic resonance spectroscopy in the metabolite pool from brain extracts of $\mathrm{PbA}$-infected mice at $6 \mathrm{dpi}$. We confirmed the increase of glutamate levels and demonstrated an enhanced release of glutamate in the synaptic cleft. Furthermore, studies of biochemical changes have demonstrated increased levels of quinolinic acid in the CSF of adults and children with CM, indicating a role of excitotoxic mechanisms in the pathogenesis of the disease $(19,20)$. Parekh et al. (10) also found an increase of glutamine levels in the metabolite pool from brain extracts of $\mathrm{PbA}$-infected mice. Taken together, these studies suggest that an imbalance in glutamate/glutamine metabolism may be relevant to $\mathrm{CM}$ pathogenesis.

In conclusion, we found that increased glutamate release is associated with neurological and behavioral symptoms in CM. These findings suggest a role for glutamate in the CNS dysfunction found in CM disease.

\section{Acknowledgments}

Research supported by CAPES, CNPq, and Rede Instituto Brasileiro de Neurociência (IBNet/FINEP), Brasil.
2. Snow RW, Guerra CA, Noor AM, Myint HY, Hay SI. The global distribution of clinical episodes of Plasmodium falciparum malaria. Nature 2005; 434: 214-217. 
3. World Health Organization (WHO). Severe falciparum malaria. Trans $R$ Soc Trop Med Hyg 2000; 94: 1-90.

4. Hunt NH, Golenser J, Chan-Ling T, Parekh S, Rae C, Potter $\mathrm{S}$, et al. Immunopathogenesis of cerebral malaria. Int $\mathrm{J}$ Parasitol 2006; 36: 569-582.

5. Meldrum BS. Glutamate as a neurotransmitter in the brain: review of physiology and pathology. J Nutr 2000; 130: 1007S-1015S.

6. Wang $\mathrm{Y}$, Qin ZH. Molecular and cellular mechanisms of excitotoxic neuronal death. Apoptosis 2010; DOI 10.1007/ s10495-010-0481-0.

7. Lau A, Tymianski M. Glutamate receptors, neurotoxicity and neurodegeneration. Pflugers Arch 2010; 460: 525-542.

8. Sanni LA, Rae C, Maitland A, Stocker R, Hunt NH. Is ischemia involved in the pathogenesis of murine cerebral malaria? Am J Pathol 2001; 159: 1105-1112.

9. Rae C, McQuillan JA, Parekh SB, Bubb WA, Weiser S, Balcar VJ, et al. Brain gene expression, metabolism, and bioenergetics: interrelationships in murine models of cerebral and noncerebral malaria. FASEB J 2004; 18: 499-510.

10. Parekh SB, Bubb WA, Hunt NH, Rae C. Brain metabolic markers reflect susceptibility status in cytokine gene knockout mice with murine cerebral malaria. Int J Parasitol 2006; 36: 1409-1418.

11. Grau GE, Piguet PF, Engers HD, Louis JA, Vassalli $P$, Lambert PH. L3T4+ T lymphocytes play a major role in the pathogenesis of murine cerebral malaria. J Immunol 1986; 137: 2348-2354.

12. Rogers DC, Fisher EM, Brown SD, Peters J, Hunter AJ, Martin JE. Behavioral and functional analysis of mouse phenotype: SHIRPA, a proposed protocol for comprehensive phenotype assessment. Mamm Genome 1997; 8: 711-713.

13. Lackner P, Beer R, Heussler V, Goebel G, Rudzki D, Helbok $\mathrm{R}$, et al. Behavioural and histopathological alterations in mice with cerebral malaria. Neuropathol Appl Neurobiol 2006; 32: 177-188.

14. Romano-Silva MA, Ribeiro-Santos R, Ribeiro AM, Gomez MV, Diniz CR, Cordeiro MN, et al. Rat cortical synaptosomes have more than one mechanism for $\mathrm{Ca}^{2+}$ entry linked to rapid glutamate release: studies using the Phoneutria nigriventer toxin PhTX2 and potassium depolarization. Biochem J 1993; 296 (Part 2): 313-319.

15. Dunkley PR, Heath JW, Harrison SM, Jarvie PE, Glenfield $P J$, Rostas JA. A rapid Percoll gradient procedure for isolation of synaptosomes directly from an $\mathrm{S} 1$ fraction: homogeneity and morphology of subcellular fractions. Brain Res 1988; 441: 59-71.

16. Nicholls DG, Sihra TS, Sanchez-Prieto J. Calcium-dependent and -independent release of glutamate from synaptosomes monitored by continuous fluorometry. $J$ Neurochem 1987; 49: 50-57.

17. de Souza JB, Riley EM. Cerebral malaria: the contribution of studies in animal models to our understanding of immunopathogenesis. Microbes Infect 2002; 4: 291-300.

18. Lacerda-Queiroz N, Rodrigues DH, Vilela MC, Miranda AS, Amaral DC, Camargos ER, et al. Inflammatory changes in the central nervous system are associated with behavioral impairment in Plasmodium berghei (strain ANKA)-infected mice. Exp Parasitol 2010; 125: 271-278.

19. Dobbie M, Crawley J, Waruiru C, Marsh K, Surtees R. Cerebrospinal fluid studies in children with cerebral malaria: an excitotoxic mechanism? Am J Trop Med Hyg 2000; 62: 284-290.

20. Medana IM, Hien TT, Day NP, Phu NH, Mai NT, Chu'ong LV, et al. The clinical significance of cerebrospinal fluid levels of kynurenine pathway metabolites and lactate in severe malaria. J Infect Dis 2002; 185: 650-656. 